\title{
A Selective Feature Matching Approach for Iris Recognition
}

\author{
Sambita Dalal \\ Department of Electronics and \\ Instrumentation Engineering \\ ITER, Siksha 'O' Anusandhan \\ University \\ Bhubaneswar, India
}

\author{
Tapasmini Sahoo \\ Department of Electronics and \\ Telecommunication Engineering \\ ITER, Siksha 'O' Anusandhan \\ University \\ Bhubaneswar, India
}

\begin{abstract}
Reliable automatic recognition of persons has long been an attractive goal of many researchers. Thus the recognition of an individual based on iris pattern is gaining more popularity due to the uniqueness of the pattern among the people which are highly stable starting from about one year past the date of birth, until death. The probability for the existence of two irises that are same has been theoretically estimated to be very high, i.e. one in $10^{72}$ which counts for the unique characterization of the iris. Although many approaches for iris recognition have been proposed by many researchers in the last few years, in this paper a selective iris feature matching method for iris recognition based on optimized wavelet decomposition of normalized iris image has been proposed. Comparing the average normalised correlation of the wavelet coefficients of optimised level and its adjacent levels improved matching is obtained, thus performing uniqueness verification of a person.
\end{abstract}

\section{General Terms}

Pattern Recognition and Analysis, Security, Proposed Matching Algorithm, Reliable Person Authentication

\section{Keywords}

Threshold; Image Morphology; Optimal level decomposition; Wavelet coefficients; Correlation; Selective iris features

\section{INTRODUCTION}

To cope with the increasing security requirement of the current commercial society, personal identification is becoming more and more important. Some traditional methods for personal identification and verification includes the token-based method that uses specific things such as ID cards or keys for enrolee authentication and the knowledgebased method that uses something you know such as password for enrolee identification. However, these methods are usually not reliable as token may be lost and knowledge may be forgotten. Also in the modern era where computers and electronics devices are more extensively used and the population of the world is increasing, there is a need for highly accurate and automatic reliable authentication technology in modern society. Therefore, in the recent few years a new advanced method for personal identification named biometrics has outfoxed the past techniques. As a promising way of authentication [1], biometrics aims to recognize a person using the physiological or behavioural characteristics such as fingerprints, face, gait, voice, iris, and retina. Iris recognition has shown a satisfactory performance in the field of classification and recognition. The human iris is the annular part between pupil and sclera, and has about 266 distinct characteristics [2] such as freckles, filaments, corneas, stripes, furrows, crypts, arching ligaments and so on. These irregularly shaped microstructures are so randomly distributed patterns which make the human iris as the most reliable biometric character recognition system. Compared with other biometric features, personal authentication based on iris recognition can obtain high accuracy due to the rich texture of iris patterns. Therefore, iris recognition has many potential applications such as access control, network security, etc. in various fields.

Tracking right through the history of iris recognition since back from the 19th century, automatic iris recognition is a newly emergent issue in biometrics. Flom and Safir [3] proposed the concept of automated iris recognition in 1987. Since then, iris recognition has been receiving many researchers' attention and a great deal of progress has been achieved in the last decade. For instance, Daugman's contribution to the automated iris recognition system is ingenious. Daugman [4,5] realized an iris recognition system in 1993 whose identification accuracy is up to $100 \%$. Daugman's iris recognition algorithm is based on the principle of the failure of a test of statistical independence on iris phase structure encoded by quadrature wavelets. Also Daugman $[5,6]$ presented an algorithm that processes the two dimensional information of the texture, thereby increasing the feature extraction time. Here Daugman used 2D Gabor filters and phase coding to generate a 2048 binary feature code for the iris. But Daugman's method was based on local features stressed on ensuring that repeated image capture produces irises on the same location within the image and they acquired the same resolution. Wildes [7] used the Gauss-Laplacian filter to decompose the iris image under the different resolution, to four resolution levels and generated iris code. Then he carried on the correlation comparison for the corresponding images, where the computation part is huge and time consuming comparatively. $\mathrm{Li}$ [8] advanced the fractional Fourier transform in iris recognition. Various other research groups such as, Ma et al, Tisse et al [9] and Zhu et al [10] has proposed a recognition system based on Haar discrete wavelets. Tan [11] adopted a texture analysis method that is the multi-channel Gabor filtering, to capture both global and local details in an iris. Eri Prasentyo Wibowo and Wisnu Sukma Moulana [12] proposed the real time iris recognition system using fusion of multiple techniques. The 
morphological operation approach such as dilation and erosion techniques were implemented on iris images. Iris localization was done using the skeleton algorithm and pupil boundary was thus detected in the process. Matching was performed using Hamming and Euclidean distance algorithms. To narrate a few more issues on the recognition system advancement, F M Wheeler et al. [13] proposed the standoff iris recognition technique. Here a dual iris approach was adopted for feature details extraction and Hamming code has been used for matching purpose. The user was thus declared as identified if one of the two irises was recognized. Also Mohammed Abdullah et al., [14] presented an algorithm of discrete wavelet transform based feature encoding for iris recognition. The acquired image was filtered using Gaussian filter for noise reduction. Using iris segmentation techniques such as the Canny edge detector and Hough transform, the iris region was segmented and then normalized. The features extracted through the wavelet transform were used to form feature vector from the enhanced iris image. XOR operator was used for Hamming code distance matching. Then the feature vector in the form of binary code was stored and utilized to create biometric smart card template for unique identification and verification purposes. The above comparative study of various existing techniques and researches has thus instigated to a great extent in developing our new approach for selective iris feature matching.

The main motivation of our work presented in the paper is selecting comparative iris matches with the subjects' iris from the feature database based on optimal decomposition of standard wavelets which provides an ease and accurate recognition technique for recognition system.

In this paper an improved iris feature matching based on multi resolution textural information to form iris signature of prominent length in the has been proposed thus improving system efficiency. The sections of the paper has been organised in the following way. The iris recognition approach is explained briefly in section II. In section III our proposed matching scheme is presented. Finally section IV discusses the experimental results and section $\mathrm{V}$ concludes the paper

\section{IRIS RECOGNITION APPROACH}

The system comprises of four stages: iris segmentation, iris normalization, feature extraction and similarity measurement. The iris database being operated in the work is of Multimedia University [15]. Our iris recognition system approach is discussed in this section.

\subsection{Binary Threshold}

The iris localization is to detect the iris inner and outer boundary between pupil and sclera from an eye image. The pupil is darker than the iris and the iris is darker than the sclera. Since the centres of both iris and pupil are close to each other, we consider the pupil centre as the approximate iris centre. Threshold $\mathrm{T}$ value calculation is done by dividing the image of an eye is into $8 \times 8$ regions. The mean intensity from each region is calculated and the lowest mean intensity value is used as a threshold. To determine the pupil location, the image of the eye $\mathrm{I}(\mathrm{x}, \mathrm{y})$ is transformed into binary image form $\mathrm{E}(\mathrm{x}, \mathrm{y})$ using the threshold $\mathrm{T}$ as in (1).

$$
E(x, y)=\left\{\begin{array}{c}
0, I(x, y) \leq T \\
255, I(x, y)>T
\end{array}\right.
$$

This process converts a gray image to binary image. The result of this process is a noisy pupil mask image as shown in figure 1 .
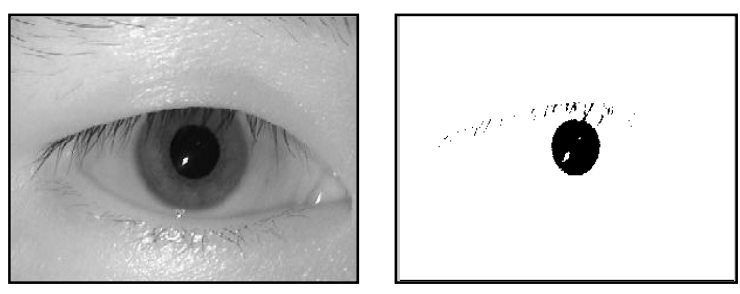

Figure 1: Sample iris image from MMU database with pupil isolation by threshold.

\subsection{Image Morphology}

In binary morphology [16,9], an image is viewed as a subset of an Euclidean space $\mathrm{E}$ or grid $\mathrm{Z}$ and $\mathrm{A}$ is a binary image in E. The basic idea in binary morphology is to probe an image with a simple, pre-defined shape, B, called 'structuring element' drawing conclusions on how this shape fits or misses the shapes in the image.

Dilation is one of the basic operations in mathematical morphology. The dilation of A by the structuring element B is defined by (2):

$$
A \oplus B=\left\{z \in E \mid\left(B^{S}\right)_{z} \cap A \neq \Phi\right\}
$$

where $\mathrm{B}^{\mathrm{S}}$ denotes the symmetric of $\mathrm{B}$, and $\mathrm{z}$ is the enlargement. If $\mathrm{B}$ has a centre on the origin, then the dilation of $\mathrm{A}$ by $\mathrm{B}$ can be understood as the locus of the points covered by B when the centre of B moves inside A. In another condition that the threshold value doesn't narrow the area, so widen the pixels with erosion technique. The erosion of the binary image A by the structuring element B is defined by (3):

$$
A \Theta B=\left\{z \in E \mid B_{z} \subseteq A\right\}
$$

where $\mathrm{B}_{\mathrm{Z}}$ is the translation of $\mathrm{B}$ by the vector $\mathrm{z}$, i.e., $\mathrm{B}_{\mathrm{Z}}$ $=\{b+z \mid b \in B\}, \forall z \in E$. When the structuring element $\mathrm{B}$ has a centre (e.g., $B$ is a disk or a square), and this centre is located on the origin, then the erosion of A by B can be understood as the locus of points reached by the centre of $\mathrm{B}$ when $\mathrm{B}$ moves inside A.

The opening of A by B is obtained by the erosion of A by B, followed by dilation of the resulting image by $\mathrm{B}$ is given by (4) as:

$$
\mathrm{A} \circ \mathrm{B}=(\mathrm{A} \ominus \mathrm{B}) \oplus \mathrm{B}
$$

The closing of A by B is obtained by the dilation of A by B, followed by erosion of the resulting structure by B is given by (5) as:

$$
\mathrm{A} \cdot \mathrm{B}=(\mathrm{A} \oplus \mathrm{B}) \ominus \mathrm{B}
$$

Generally, the erosion and dilation smooth the boundaries of objects without significantly changing their area. Opening and closing are to smooth thin projections or gaps.

Mathematical binary morphology is performed on threshold binary image to obtain an image containing accurate and black pupil of eye only, so that center of the pupil can be found with ease. After performing dilation and erosion on the noisy pupil, extracted pupil of the sample iris is shown below in figure 2 . 


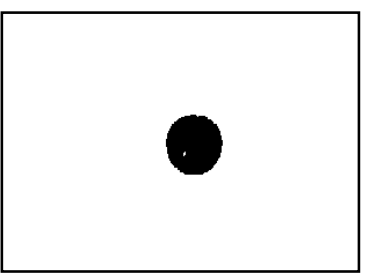

Figure 2: Pupil extracted after morphological processing.

\subsection{Iris Segmentation}

The next step involves determining the centre of the pupil [17]. This is done by finding the row and column having the maximum number of pixels of gray level ' 0 ', horizontally, vertically and diagonally. The average of the pixel values of the three centre positions obtained corresponds to the centre of the pupil. The pupil radius is now obtained. This pupil centre also has been assumed as iris centre and thus iris radius is obtained. The pupil radius is not more than 0.7 and neither less than 0.1 times of the iris radius.

Both the iris and pupil circular plots on eye used to obtain concentric iris boundaries. Thus the iris is extracted from the eye image, that is the disturbing features like eyelids \& eyelashes are eliminated to the maximum possible extent in the preceding processes and then process of normalization is carried out.

\subsection{Iris Normalization}

Now the segmented iris is normalised through homogenous rubber sheet model devised by Daugman [18] remaps each point within the iris region to a pair of polar coordinates. The remapping of the iris region from $(x, y)$ Cartesian coordinates to the normalized non-concentric polar representation $(r, \theta)$ where $r$ is on the interval $[0,1]$ and $\theta$ is angle $[0,2 \pi]$ is modeled as defined by (6) and the resulting image is shown in figure 3 .

$$
\mathrm{I}(\mathrm{x}(\mathrm{r}, \theta), \mathrm{y}(\mathrm{r}, \theta)) \rightarrow \mathrm{I}(\mathrm{r}, \theta)
$$

with

$$
\begin{aligned}
& x(r, \theta)=x_{p}(\theta) \cdot(1-r)+x_{i}(\theta) \cdot r \\
& y(r, \theta)=y_{p}(\theta) \cdot(1-r)+y_{i}(\theta) \cdot r
\end{aligned}
$$

where $I(x, y)$ is the iris region image, $(x, y)$ are the original Cartesian coordinates, $(r, \theta)$ are the corresponding normalized polar coordinates, and $\left(\mathrm{x}_{\mathrm{p}}, \mathrm{y}_{\mathrm{p}}\right)$ and $\left(\mathrm{x}_{\mathrm{i}}, \mathrm{y}_{\mathrm{i}}\right)$ are the coordinates of the pupil and iris boundaries along the $\theta$ direction.

\section{PROPOSED TECHNIQUE}

Our work detailed in this paper focuses on selecting the irises whose features are to be matched with an enrollee/imposter for identification and verification attempts. But prior to our work, one can date back to the history of iris recognition algorithms, where many researchers have adopted various multi-classifiers and iris matchers' processes to obtain accurate person identification and verification through. To recall a few of iris recognition approaches [19] and their matching methods are discussed. Daugman (1993, 2003, 2007), Schuckers et al. (2005), Liu et al.(2007) and Monro et al. (2005) obtained good recognition rate with Hamming distance matching of iris codes. Wildes (1997) performed normalized correlation based iris matching which was time consuming. Ma et al. (2004) used weighted Euclidean distance for local features matching. Also classifiers such as K Nearest Neighbor, Support Vector Machines and soon have been implemented widely in the last decade for recognition purpose. The analysis of the existing techniques proves useful while comparing the advantages of our proposed selective feature technique with those of others. Algorithm for the proposed technique has been given in sub-section $\mathrm{D}$. and the technique is discussed below.

\subsection{Optimized DWT Decomposition}

The texture details [20] in the iris region can be analyzed at different resolutions using its multi-scale wavelet decomposition. Wavelet analysis is such an approach in which window with variable-sized regions is used. In wavelet analysis, we have approximations and details coefficients. The approximations are the high-scale, low-frequency components of the signal. The details are the low-scale, high-frequency components. Wavelets give a better signal representation using multi-resolution analysis with balanced resolution at any time and frequency. A multi-resolution analysis of the space $L^{2}(\Re)$ consists of a sequence of the nested subspaces as given by: $\{0\} \subset V_{0} \subset V_{1} \subset V_{2} \ldots \subset V_{n} \subset V_{n+1} \subset \ldots \subset L^{2}(\Re)$.
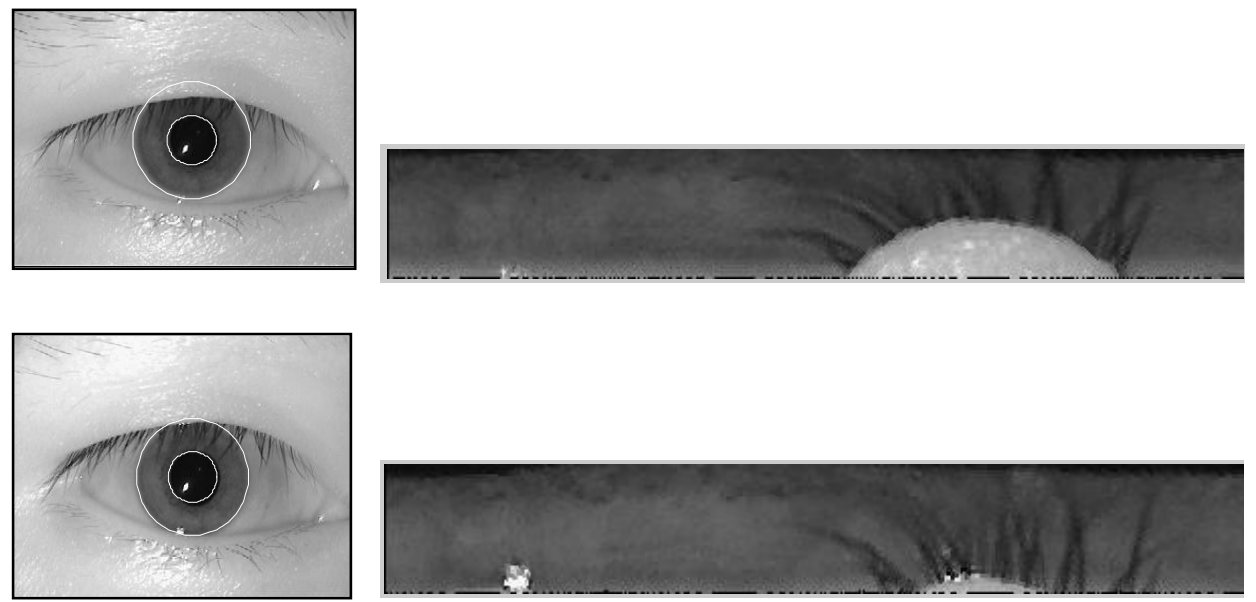

Figure 3: Localized iris unwrapping to normalized polar coordinates of two images of same iris in varying conditions. 
As it is computationally impossible to analyze a signal using all wavelet coefficients, we use the affine system for some real parameters $a>1, b>0$. The corresponding discrete subset of the half plane consists of all the points $\left(a^{m}, n a^{m} b\right)$ with integers $m, n \in \mathrm{Z}$. The corresponding baby wavelets are now given as (7).

$$
\Psi_{m, n}(t)=a^{-m / 2} \Psi\left(a^{-m} t-n b\right)
$$

The approximate and detail coefficients obtained by discrete wavelet transform [21,22] of image $f(x, y)$ of size $\quad M x ~ N$ is then given in (8) with scaled and translated basis functions are given as in (9).

$$
\begin{gathered}
W_{\Phi}\left(j_{0}, m, n\right)=\frac{1}{\sqrt{M N}} \sum_{x=0}^{M-1} \sum_{y=0}^{N-1} f(x, y) \Phi_{j_{0}, m, n}(x, y) \\
W_{\Psi}^{i}(j, m, n)=\frac{1}{\sqrt{M N}} \sum_{x=0}^{M-1} \sum_{y=0}^{N-1} f(x, y) \Psi_{j, m, n}^{i}(x, y), i=\{H, V, D\}
\end{gathered}
$$

where index $i$ identifies the directional wavelets and $\mathrm{j}_{0}$ is an arbitrary starting scale and $\mathrm{j} \geq \mathrm{j}_{0}$.

$$
\begin{gathered}
\Phi_{j, m, n}(x, y)=2^{j / 2} \Phi\left(2^{j} x-m, 2^{j} y-n\right) \\
\Psi^{i}{ }_{j, m, n}(x, y)=2^{j / 2} \Psi^{i}\left(2^{j} x-m, 2^{j} y-n\right), i=\{H, V, D\}
\end{gathered}
$$

The optimum sub band decomposition level of the approximation and detailed coefficients is obtained through evaluation of normalized correlation given by (10).

$$
\frac{\sum_{i=1}^{m} \sum_{j=1}^{n}\left(l_{k 1}[i, j]-\mu_{1}\right)\left(l_{k 2}[i, j]-\mu_{2}\right)}{m n \sigma_{1} \sigma_{2}}
$$

where $p_{1}$ and $p_{2}$ are two segmented iris images of size $m \times n$, $\mathrm{k}$ is the number of decomposed sub-band, $\mu_{1}$ and $\sigma_{1}$ are the mean and standard deviation of $\mathrm{p}_{1}$, and $\mu_{2}$ and $\sigma_{2}$ are the mean and standard deviation of $\mathrm{p}_{2}$. Normalized correlation is employed for evaluating the degree of similarity and has been shown to give accurate results. The average of normalised correlation of approximate coefficients was calculated at each level. Here we obtain the optimum level of decomposition that can be allowed is fifth level. The approximate coefficient image till sixth level decomposition is shown in figure 4 .

\subsection{Feature Database Creation}

The database used in the experimentation consists of 450 different iris images from MMU iris database version 1.0 [15]. Size of each database iris image is $320 \times 240$. In the database there are 90 different subjects. Each subject has contributed to five different images of same eye. Thus there are a total of $450(90 \times 5)$ images in the database. To calculate the signature of iris we have used local energy given by (11), taking window of size $5 \times 5$ and computed standard deviation $\sigma$ given by (12) of each sub-band. To avoid complexity in database handling and reduce computational time, feature database was created with three level of decomposition $[21,23]$. Thus the signature vector size of database is of $450 \mathrm{x}$ $8 \times 3$ and for query image it is of size $1 \times 8 \times 3$.

$$
\text { Energy }=\sum_{i=0}^{m-1} \sum_{j=0}^{n-1}\left[l_{k}(i, j)\right]^{2}
$$

$$
\text { Standard deviation }=\sqrt{\frac{1}{m \times n} \sum_{i=1}^{m} \sum_{j=1}^{n}\left(l_{k}(i, j)-\mu\right)^{2}}
$$

where $\mathrm{k}$ is the $\mathrm{k}^{\text {th }}$ decomposed sub-band and $\mu$ is the mean.

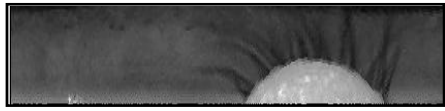

(a) Original Iris Image

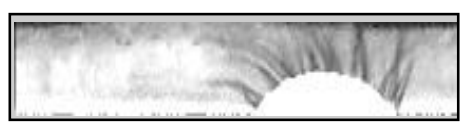

(b) Level 1

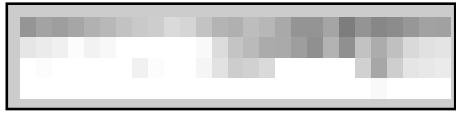

(e) Level 4

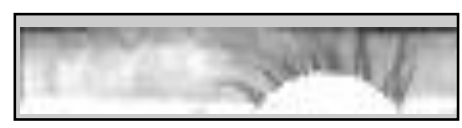

(c) Level 2

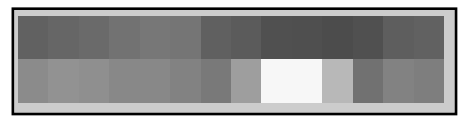

(f) Level 5

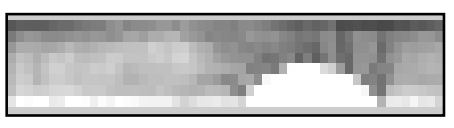

(d) Level 3

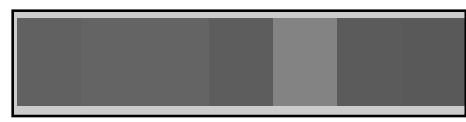

(g) Level 6

Figure 4: (a) Original normalized iris (b)-(g) approx coefficients of first to sixth sub-band decomposition levels 


\subsection{Selective Matching}

The degree of match between the acquired image and images from the database is evaluated using normalized correlation between them. Those selective iris from the database whose average normalized correlation of approximate coefficients with that of the query iris image at the next level of optimum is strong, that is near to one, they form similar iris set to be considered for Euclidean distance matching. If correlation between them decreases they are irises of different eyes.

The global features, that is energy and standard deviation represents the texture of iris. Compared with the stored template of iris, the Euclidean distance between the two corresponding global iris features vector is obtained. The Euclidean distance gives a measure of how similar a collection of values is between two templates as given in (13). Zero distance implies a perfect match, and signature tends towards mismatch as the distance increases.

$$
D^{\text {euclidean }}(x, y)=\sqrt{\sum_{i=0}^{N}\left(x_{i}-y_{i}\right)^{2}}
$$

where $x_{i}$ and $y_{i}$ are $i^{\text {th }}$ feature vector of test/query iris and database iris template respectively.

\subsection{Proposed Algorithm}

Input: Query eye image

Output: Persons' iris identification

Level $\mathrm{L}=0$

Step 1: Read the eye image

Step 2: Iris localization and segmentation

Step 3: The Daubechies wavelet is applied, $\quad$ Level $=\mathrm{L}+1$

Step 4: Evaluate normalised correlation of the test or query iris with database irises

Step 5: If average not less than 0.9, perform step 3-5 again

Step 6: Level L is the optimum level

Step 7: Form the signature vector of each iris image

Step 8: Compute normalised correlation NC between two iris images at $\mathrm{L}+1$ sub-band level

Step 9: Select irises with value more than 0.9 as a Match

Step 10: Compute Euclidean distance with selected irises feature (size is 24 ) vector

Step 11: Match/No match decision is obtained from the distance obtained in step 10

\section{IMPLEMENTATION RESULTS}

We perform experiments in Matlab version 7.5 [24] to evaluate the performance of the proposed algorithm over iris images supplied by MMU version1.0 [15]. In this images database, a few images with noise, eyelids, eyelash, \& illumination interferences are involved. The test eye images database consists of 90 distinct iris images. The algorithm was applied for each image in the database. Results obtained by the proposed method are much interesting. We extract the features and form iris signature from approximate and detail coefficients of the segmented iris at various decomposition sub-bands of discrete wavelet transform
The decision making processes are based on the goodness of match of correlation coefficient measurements. Normalized correlation is employed for evaluating the degree of similarity and has been shown to give accurate results. The normalized correlation coefficients was obtained for a given query iris signature with those of all database iris signatures at each decomposition levels until the value at some level decreases drastically to a considerable amount. Here we obtain the optimum level of decomposition that can be allowed. To its next level correlation among distinct irises reduces. Finally we decide whether the acquired image and image from the database are derived from the same iris, based on the degree of match at the next sub band level to the optimised sub band level. An example of the result obtained from our experiment is as shown in the table 1 and table 2 below. A comparative study of average normalized correlation value of a test iris with the same irises is presented in table 1 and with that of other database irises is presented in table 2, at various decomposition levels.

Table 1. Average Normalized Correlation Result of the Test IRIS 1A with same person Irises from the database.

\begin{tabular}{|c|c|c|c|}
\hline $\begin{array}{c}\text { Average } \\
\text { Normalized } \\
\text { Correlation } \\
\text { Values }\end{array}$ & IRIS 1A & IRIS 1B & IRIS 1C \\
\hline Level 1 & 1.0000 & 1.0000 & 1.0000 \\
\hline Level 2 & 1.0000 & 0.9999 & 0.9999 \\
\hline Level 3 & 1.0000 & 0.9998 & 0.9998 \\
\hline Level 4 & 1.0000 & 0.9996 & 0.9994 \\
\hline $\begin{array}{c}\text { Optimal } \\
\text { Level 5 }\end{array}$ & 1.0000 & 0.9985 & 0.9970 \\
\hline Level 6 & 1.0000 & 0.9672 & 0.9907 \\
\hline
\end{tabular}

Table 2. Average Normalized Correlation Result of the Test IRIS 1A with some other persons' Irises from the database.

\begin{tabular}{|c|c|c|c|c|}
\hline $\begin{array}{c}\text { Average } \\
\text { Normalized } \\
\text { Correlation } \\
\text { Values }\end{array}$ & IRIS 2A & IRIS 2B & IRIS 3A & IRIS 3B \\
\hline Level 1 & 0.9999 & 0.9999 & 0.9999 & 0.9999 \\
\hline Level 2 & 0.9997 & 0.9998 & 0.9997 & 0.9996 \\
\hline Level 3 & 0.9987 & 0.9994 & 0.9988 & 0.9991 \\
\hline Level 4 & 0.9940 & 0.9974 & 0.9970 & 0.9965 \\
\hline $\begin{array}{c}\text { Optimal } \\
\text { Level 5 }\end{array}$ & 0.9607 & 0.9785 & 0.9712 & 0.9799 \\
\hline Level 6 & 0.7258 & 0.6010 & 0.5129 & 0.4399 \\
\hline
\end{tabular}


We obtained that the average normalised correlation of approximate coefficients of irises of same eye lies above 0.9 and reduces to a visible extent for distinct irises at the next decomposition sub-band of the derived optimisation level that is sixth level. This result is observed to a remarkable extent, for maximum subjects' eye images in the database. We obtain a few probable set of matching irises. These selective iris features are employed for distance matching. Thus the Euclidean distance calculation of the query iris signatures with those resulting selected set of probable irises was performed. The signature vector size of database is of $450 \mathrm{x}$ 24 and for query image it is of size $1 \times 24$, where 24 indicates number of features taken. Zero distance implies a perfect match and mismatch improves as the distance increases.

\section{CONCLUSION}

The proposed selective iris matching algorithm is used to improve reliability of the iris recognition system. In this paper, the optimum sub band decomposition level obtained through evaluation of normalized correlation which is found to improve recognition system accuracy noticeably. Then value of statistical parameters efficiently identifies the subject's iris as double strainer process is achieved. The decision-making is made simpler and the robustness of the recognition system also increases. The selective iris set matching thus enhances the matching criterion and can reduce the False Acceptance Rate of imposter and False Rejection Rate of enrolee in the recognition system considerably. Results achieved in this paper are promising and some additional researches will be performed in the future with variety of iris images taken under varying conditions.

\section{ACKNOWLEDGMENTS}

Our thanks to the Iris Recognition Research Group and the MMU iris database that is Multimedia University database for sharing their database of iris images. Also we present our thanks to Sanjukta Prusty, Biswajit Biswal and Sandeepta Dalal for their kind cooperation towards the completion of the work.

\section{REFERENCES}

[1] Bowyer, K.W., Hollingsworth, K., Flynn, P., “ Image understanding for iris biometrics: a survey", Comp. Vision and Image Understanding 110 (2008), pp: 281307.

[2] Dobes, M., Machala, L., Tichavsky, P., Pospisil, J., "Human eye iris recognition using the mutual information", International Journal for Light and Electron Optics, No. 9, 2004, pp: 399-404.

[3] Flom, L., Safir, A., "Iris recognition system", US Patent no. 4641 394, 1987.

[4] Daugman, J., "The importance of being random: Statistical principles of iris recognition", Pattern Recognition, Vol. 36, No. 2, 2003, pp: 279-291.

[5] Daugman, J., "High Confidence Visual Recognition of Persons by a Test of Statistical Independence", IEEE Trans. On Pattern Analysis and Machine Intelligence, Vol. 15, No.11, 1993, pp: 1148-1161.

[6] Daugman, J., "How iris recognition works", Proceedings of 2002 International Conference on Image Processing, Vol.1, 2002.

[7] Wildes, R., "Iris recognition: an emerging biometric technology", Proc. IEEE, 1997, 85(9), pp: 1348-1363.
[8] Yu, Li., "Iris Verification Based on Fractional Fourier Transform", Proceedings of the International Conference on Machine Learning and Cybernetics, Beijing, Nov 2002, pp: 1470-1473.

[9] Tisse, C.L., Torres L., and Robert M., "Person Identification based on iris patterns", Proceedings of the $15^{\text {th }}$ International Conference on Vision interface, 2002.

[10] Zhu, Y., Tan T., and Yusag, "Biometric Personal Identification based on Iris Patterns", Pattern recognition, 15th Internal Configuration, vol. 2, 2004, pp: 801-804.

[11] Ma, Li., Tan, Tieniu., "Personal Identification Based on iris Texture Analysis", IEEE Transaction on pattern analysis and machine intelligence, Vol. 25, No. 12, 2003, pp: 519-1533.

[12] Wibowo, Eric Prasetyo., Maulana, Wisnu Sukma., IEEE International Conference on Signal Processing, 2009, pp: 98-102.

[13] Wheeler, F M., Perera, A G A., Abramovich G., Bing Yu. and Tu P H., "Stand-Off Iris Recognition System", Second IEEE International Conference on Biometric Theory, Applications and Systems, 2008, pp: 1-7.

[14] Abdullah, Mohammed A M., Al-Dulaimi, F H A., AlNuaimy, Waleed. and Al-Ataby Ali., "Smart Card with Iris Recognition for Hifh Security Access Environment," IEEE International Conference on Biomedical Engineering, 2011, pp: 382-385.

[15] MMU Iris Database, http://pesona.mmu.edu.my/ ccteo/

[16] Gonzalez, Woods, R.C. dan., R.E., Digital Image Processing $2^{\text {nd }} /$ ed., Prentice-Hall. Inc., Upper Saddle River, New Jersey, 2002.

[17] Ganeshan, B., Theckedath, D., Young, R., Chatwin, C., "Biometric iris recognition system using a fast and robust iris localization and alignment procedure", Optics and Lasers in Engineering, Vol. 44, 2006, pp: 1-24.

[18] Masek, L.,"Recognition of Human Iris Patterns for Biometric Identification", Thesis, School of Computer Science and Software Engineering, University of Western Australia, 2003.

[19] Roy, K ., Bhattacharya, P., Suen, Ching Y., "Towards nonideal iris recognition based on level set method, genetic algorithms and adaptive asymmetrical SVMs" Elsevier Engineering Applications of Artificial Intelligence, Vol. 24, 2011, pp: 458-475.

[20] Nabti, M., Bouridane, A., "An effective and fast iris recognition system based on a combined multiscale feature extraction technique”, Pattern Recognition, Vol. 44, 2008, pp: 868-879.

[21] Birgale, L.V., Kokare, M., "Iris recognition using discrete wavelet transform”, IEEE Trans. an International Conference on Digital Image Processing(ICDIP), 2009, pp: 147-151.

[22] Wheeler, F M., Perera, A G A., Abramovich G., Bing Yu. and Tu P H., "Stand-Off Iris Recognition System", Second IEEE International Conference on Biometric Theory, Applications and Systems, 2008, pp: 1-7.

[23] Zhang, P., Li, De., Wang, Qi., "A Novel Iris recognition Method Based on Feature Fusion", IEEE Proceedings of the Third International Conference on Machine Learning and Cybernetics, Shanghai, August 2004, pp:3661-3665.

[24] MATLAB The Language of Technical Computing, The Mathworks. 